\title{
PHOTODEGRADATION OF HEXAMETHYLENETETRAMINE BY VUV AND ITS RELEVANCE FOR CN AND HCN EXTENDED SOURCES IN COMETS
}

\author{
Hervé Cottin, Souleyman Bachir, François Raulin and Marie-Claire Gazeau \\ Laboratoire Interuniversitaire des Systèmes Atmosphériques (LISA), UMR 7583 CNRS, Université Paris 7 and Paris 12 , \\ C.M.C., 61 Avenue du Général de Gaulle, 94010 Créteil Cedex, France
}

\begin{abstract}
This paper presents our first experimental results on the photodegradation at 147 and $122 \mathrm{~nm}$ of hexamethylenetetramine (HMT). This molecule, suspected to be present on cometary grains, could he responsible for extended sources of CN and HCN in comets. We show that if HMT is quite resistant to direct photolysis under vacuum, interactions with water vapor photoproducts, which could happen in inner comae, lead to a much more efficient degradation with formation of $\mathrm{HCN}, \mathrm{NH}_{3}$ and other $\mathrm{N}$ bearing molecules. Thus HM'T could act as a parent or grand parent molecule for observed extended sources. However, additional source with greater degradation efficiency is certainly required to account observations in outer comae. (C) 2002 COSPAR. Published by Elsevier Science Ltd. All rights reserved.
\end{abstract}

\section{INTRODUCTION}

The nature of organic components of interstellar dust and comets is critical to understanding the chemical cvolution of materials from the interstellar medium to the solar system. A classical laboratory approach to this problem is to start with various ice mixtures at temperature of $10 \mathrm{~K}$ and to photolyze with vacuum ultraviolet or irradiate with charged particles, or simply warm the ices, simulating the processing of ice-mantled dust in molecular clouds. When warmed to room temperature, these processed mixtures leave a residue which is called " yellow stuff » and which is generally presumed to be a close analog of the organic mantles of dust in molecular clouds (see for instance : (Allamandola et al., 1988; Briggs ct al., 1992), or (Cottin et al., 1999) for a review on that subject).

Among the complex organic refractory molecules that have been synthesized during cometary and interstellar ice analog experiments, polyoxymethylene (POM - $\left(\mathrm{CH}_{2}-\mathrm{O}\right)_{n}$ ) and hexamethylenetetramine (HMT $-\mathrm{C}_{6} \mathrm{H}_{12} \mathrm{~N}_{4}$ ) (see Figure 1) seem to be of prime interest. POM and polymers of the same family have been detected when ice mixtures with formaldehyde and ammonia are slowly warmed to room temperature, without any photolysis or ion irradiation processing (Schutte ct al., 1993a; Schutte et al., 1993b). After UV photolysis of mixtures such as $\mathrm{H}_{2} \mathrm{O}: \mathrm{CH}_{3} \mathrm{OH}: \mathrm{CO}: \mathrm{NH}_{3}(10: 5: 1: 1)$, POM-like polymers have also been detected, but HMT represents $\sim 60 \%$ of the organic residue at $300 \mathrm{~K}$ (Bernstein et al., 1995). Thus, its looks like the presence of an important amount of POM is a good indicator for thermal processing of ices, while HMT plays the same role concerning UV photolysis. HMT is of particular interest since it forms amino acids when acid-hydrolyzed (Wolman et al., 1971).

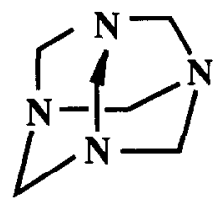

Fig. 1: Hexamethylenetetramine

Based on the interstellar dust model of comets as consisting of more or less reprocessed aggregated interstellar silicate grains with organic mantles, it was predicted that the comet dust would not only be rocky material (silicates), but have a significant fraction in the form of refractory organics; i.e. organics which are non-volatile with respect to the ices (Greenberg, 
1982). This prediction was supported by the mass spectrometry analysis performed on the VEGA 1\&2 and GIOTTO spacecraft, scnt to fly by Comet Halley (Kissel and Krueger, 1987; Mitchell et al., 1992). However, even with this basic confirmation of the idea of organic mantles, one puzzling feature of comet dust is the source of the distributed CO (DiSanti et al., 1999; DiSanti et al., 1997; Eberhardt et al., 1987), $\mathrm{H}_{2} \mathrm{CO}$ (Biver et al., 1999; Bockelée-Morvan et al., 1998; Lis et al., 1997; Meier et al., 1993), CN (Woodney et al., 1998) and HCN (Veal et al., 2000) in cometary comae. It is likely that their formation results from the thermal and/or photodegradation of refractory material on the outflowing dust. It has been shown that the thermal and photodegradation of polyoxymethylcne on cometary grains could be a good explanation of formaldehyde in comet Halley (Cottin, 1999; Cottin et al., 2001). Since HMT synthesis is favored in photolyzed ice mixtures, its presence is likely on cometary grains, and its degradation could be a source of $\mathrm{N}$ bearing molecules, and thus may be a source for $\mathrm{HCN}$ and $\mathrm{CN}$ in comae.

\section{$\mathrm{CN}$ and HCN extended sources in comets, and relevance of HMT as potential parent molecule}

An extended source of $\mathrm{CN}$ radical has been detected in comet Halley, and could have for origin the degradation of larger molecules on grains (Klavetter and A'Hearn, 1994). Moreover, in most comets, the observed HCN is not enough to account for $\mathrm{CN}$ (Crovisier and Schloerb, 1991). Hale-Bopp observation at important heliocentric distances (>2.9 AU) did not show any evidence for such a process (Rauer et al., 1997), but that far from the sun, the organic component of grains might be too cold or not irradiated enough to release $\mathrm{CN}$. On the other hand, for smaller heliocentric distance, $\mathrm{HCN}$ and $\mathrm{CN}$ distribution maps are not compatible, which implies another parent molecule for the radical (Woodney et al., 1998). The abundance of the other detected potential parent molecules ( $\mathrm{HNC}, \mathrm{CH}_{3} \mathrm{CN}, \mathrm{HC}_{3} \mathrm{~N}$ ) is not sufficient to solve this problem.

Concerning HCN, (Veal et al., 2000) have shown that its distribution in the coma of Hale-Bopp presents discrepancies with the one that would result from a direct sublimation from nucleus. The authors propose that a release of HCN from icy grains could explain observation. But in this case, we think that most of the other detected volatile molecules, subliming with the water, would present the same phenomenon, which has not been yet proved. Therefore, the process responsible of the extended source phenomenon is certainly a slow degradation of the refractory organic component of grains.

HMT could be such a parent molecule. Indeed, photolysis of HMT in water and argon matrices at $\sim 12 \mathrm{~K}$ have already been performed (Bernstein et al., 1994). It leads to the production of nitriles (RCN), isonitriles (RNC), and other N bearing compounds. In this paper, we present results concerning HMT photodegradation at room temperature, and more precisely the study of gaseous molecules released during degradation. This process simulates the action of solar UV on cometary grains, in order to conclude if HMT can be at the origin of HCN and $\mathrm{CN}$ extended sources. HMT has been photolyzed under vacuum, and with water vapor to simulate the photochemical environment in comae.

\section{EXPERIMENTAL}

\section{Photochemical Reactor}

The experimental set-up has been described in details in (Cottin et al., 2000). It mainly consists in a pyrex reactor topped by a UV lamp (Fig. 2). The reactor is equipped with a vacuum stopcock leading to the analysis system (or to a cold trap), and another connection to a secondary vacuum pump (Turbomolecular pump, Balzers). A vacuum better than $10^{-4} \mathrm{mb}$ can be reached during pumping. The photochemical reactor has a double wall for circulation of a thermostated liquid necessary to operate at a constant temperature.

The UV irradiation system is composed of a lamp filled with an appropriate gas or gas mixture according to the emitted wavelength: $5.5 \mathrm{mb} \mathrm{H}_{2}(2 \%)$ in $\mathrm{He}(>99.99 \%$ - Linde - France) for a $122 \mathrm{~nm}$ emission (Lyman $\alpha$ ), and $0.3 \mathrm{mb}$ Xe ( $99.99 \%$ - Linde - France) for $147 \mathrm{~nm}$. Discharge in the lamp is initiated with a Tesla coil and the gas is excited by a microwave generator (Somelec France) with a power of $200 \mathrm{~W}$ at $2450 \mathrm{MHz}$. The pressure of the gas in the lamp is sct at a levcl allowing to maintain a stable discharge. Titanium wires heated by an electric current can be used as efficient getters to purify Xenon during $147 \mathrm{~nm}$ experiments. The window between the lamp and the reactor is made of $\mathrm{MgF}_{2}$. The photon flux of the two lamps determined by chemical actinometry are $f_{122 n m} \sim 210^{15}$ photons. $s^{-1}$ and $f_{147 \mathrm{hm}} \sim 10^{16}$ photons. $\mathrm{s}^{-1}$.

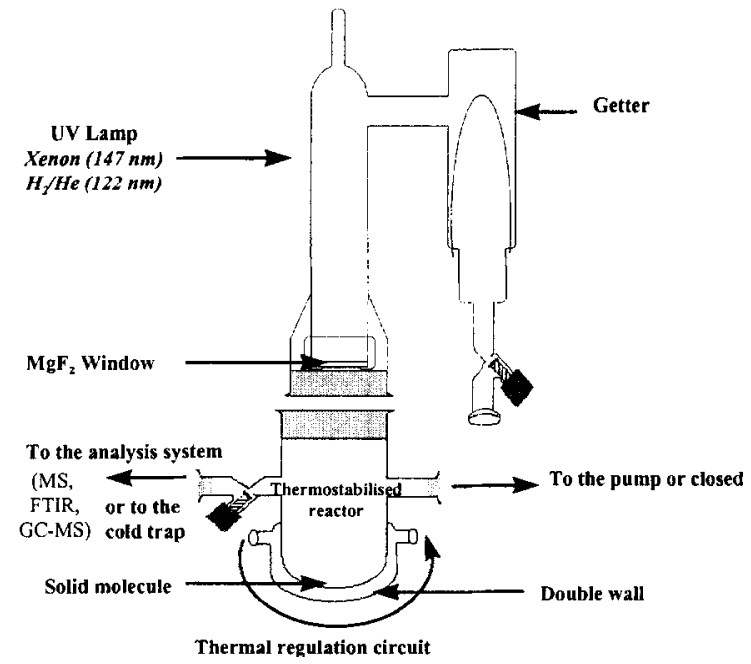

Fig. 2: Photochemical reactor and UV lamp 


\section{Chemical}

We have used commercial hexamethylenetetramine ( $>99.5 \%$, Fluka). The molecule is deposited at the bottom of the reactor and irradiated at different wavelengths after several days of pumping. Irradiation experiments have been performed at room temperature. HMT vapor pressure is negligible and no spontaneous thermal degradation has been observed.

Two kinds of experiments have been performed : HMT photolysis under vacuum, and photolysis of HMT with $0.1 \mathrm{mb}$ of water vapor.

\section{Analysis}

Preliminary chemical analysis of the gaseous mixture resulting from the photolyzed HMT has been carried out using a mass spectrometer (Leybold Inficon - Transpector). MS experiments have been performed at $5.10^{-5} \mathrm{mb}$, between 1 and 200 AMU with a resolution of $1 \mathrm{AMU}$. This lechnique is very sensitive, but since there is no separation of the different products before analysis, it can only give hints of the presence of a compound if one refers to the major ions detected. MS analysis can only be performed when HMT is irradiated under vacuum : presence of water vapor $(0.1 \mathrm{mb})$ would saturate the MS detector (quadripole), which is usable for pressure $<10^{-4} \mathrm{mb}$.

The photodegradation products can also be concentrated in a cold trap at $\sim 70 \mathrm{~K}$ (liquid nitrogen). The concentration is carried during irradiation when HMT is degraded under vacuum, or after irradiation in the presence of water vapor. When the trap is warmed up at room temperature, the resulting gaseous sample can be analyzed by FTIR (Perkin Elmer, 1710), or injected into a GC (Varian 3400), through a column MXT-1701 (Restek, $40^{\circ} \mathrm{C}$, head pressure : 15 psi). The detector is a ion trap mass spectrometer (Finnigan - Varian) ranging from 1 to $650 \mathrm{amu}$, with a resolution of 1 AMU.

\section{RESULTS}

\section{HMT irradiation under vacuum}

\section{MS analysis}

Irradiations have been performed at 122 and $147 \mathrm{~nm}$. No production of gaseous fragment have been detected during irradiation, including the 26 amu fragment that would suggest a production of $\mathrm{CN}$ radical resulting from HMT photodegradation.

\section{FTIR analysis}

In order to obtain a better detection, a cold trap has been used to concentrate photofragments for an FTIR analysis. Two hours irradiations have been performed at $147 \mathrm{~nm}$.

Fig. 3 shows $\mathrm{HCN}$ detection by its signature at $713 \mathrm{~cm}^{-1}$. Its amount is too low to be quantified. Some $\mathrm{CO}_{2}$ is also detected by its signature centered at $668 \mathrm{~cm}^{-1}$, and its band at $720 \mathrm{~cm}^{-1}$. Its presence is due to an atmospheric contamination in the spectroscopic compartment and also to impurities in the industrial $\mathrm{N}_{2}$ that is used to adjust the total pressure in the analysis cell at about $800 \mathrm{mb}$ (an augmentation of the total pressure in the analysis cell broadens the IR bands of most compounds, and allows a better detection sensitivity).

No photofragment has been detected at $122 \mathrm{~nm}$, probably because the lamp flux is significantly lower than for the $147 \mathrm{~nm}$ lamp.

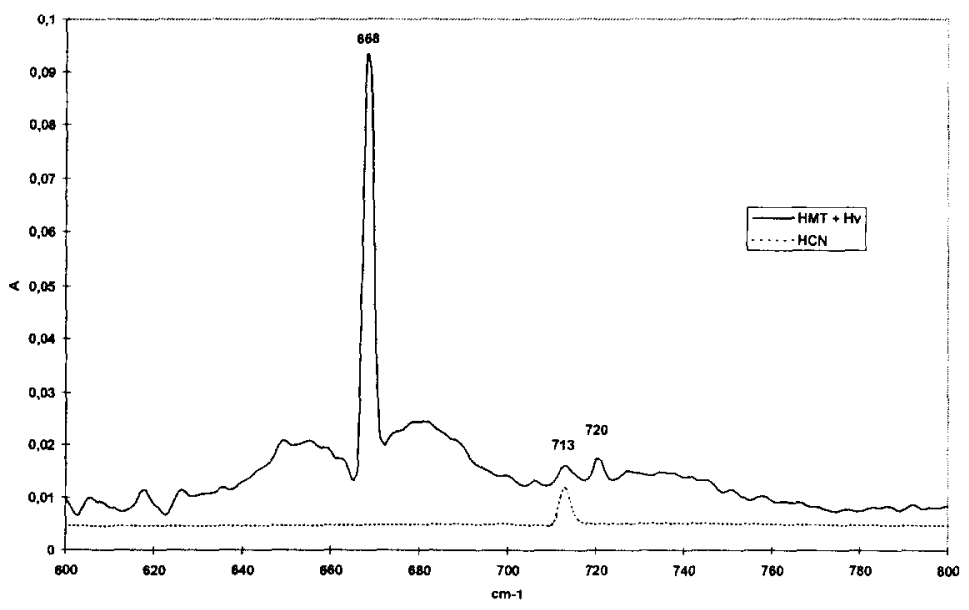

Fig. 3: Continuous line: Infrared spectrum of the gaseous phase resulting from HMT photodegradation, after 2 hours of concentration at $147 \mathrm{~nm}$, between 600 and $800 \mathrm{~cm}^{-1}$. Dotted line: $\mathrm{HCN}$ infrared spectrum. For both spectra : optical path: $10 \mathrm{~cm}$; resolution: $2 \mathrm{~cm}^{-1}$. Pressure completed at $800 \mathrm{mb}$ with nitrogen. 


\section{FTIR analysis}

6 hours irradiations of HMT at $147 \mathrm{~nm}$ have been performed with $0.1 \mathrm{mb}$ of water vapor. This pressure is not relevant of cometary environment, which is about $10^{-4} \mathrm{mb}$ near the nucleus. Our aim is not to simulate the coma, but to study a chemical mechanism. The pressure has been chosen in order (1) that most of the UV flux reaches the HMT ( $99 \%)$, and (2) obtain a process efficient enough (i.e. enough water) to allow detection of photoproducts. A resulting IR spectrum is presented on Figure 4. Again, $\mathrm{HCN}$ is detected by its signature at $713 \mathrm{~cm}^{-1} . \mathrm{NH}_{3}$ is also identified by its signatures at 967 and $929 \mathrm{~cm}^{-1}$. Amounts of $\mathrm{HCN}$ and $\mathrm{NH}_{3}$ detected decrease from an experiment to the other. This is due to a decrease of the lamp flux induced by the formation, on the window of the lamp, of an organic polymer, which absorbs the UV photons. Therefore, our experiments are not reproducible because the photonic flux cannot be controlled. No compound have been detected for irradiation at $122 \mathrm{~nm}$.

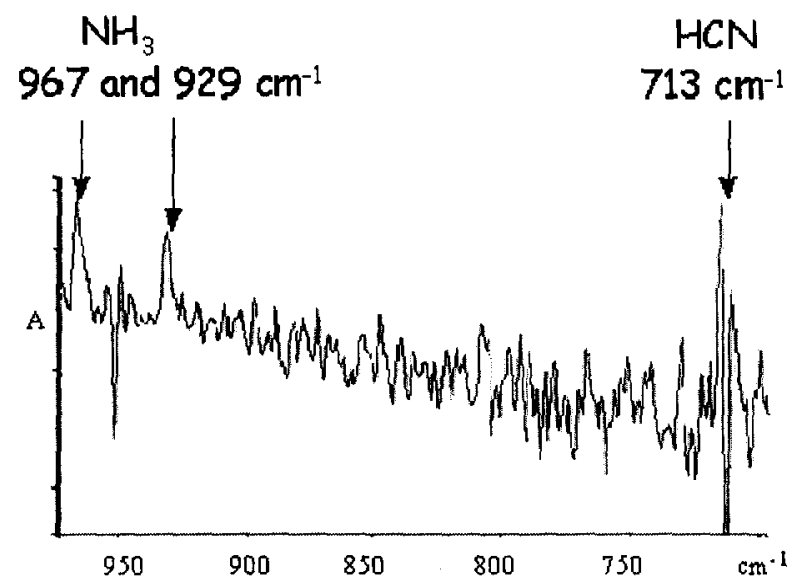

Fig. 4: Infrared spectrum of the gaseous phase resulting from HMT photodegradation, after 6 hours of irradiation at $147 \mathrm{~nm}$, between 700 and $1000 \mathrm{~cm}^{-1}$. Optical path : 10 $\mathrm{cm}$; resolution: $2 \mathrm{~cm}^{-1}$. Pressure completed at $800 \mathrm{mb}$ with nitrogen.

A rough estimation of the production quantum yields for both detected compounds can be achieved (how many molecules are produced for one photon in our photochemical system : $\mathrm{HMT}+\mathrm{H}_{2} \mathrm{O}$ ). Considering the first experiment, for which the lamp flux is assumed at its highest level (measurement by chemical actinometry before irradiations), one can write :

$\frac{d[H C N]}{d t}=\Sigma$ sources $-\Sigma \sin k s=\frac{F l u x^{*} \Phi_{h o v}}{V}-\left(J_{H O N} *[H C N]\right)$

With :

[HCN] : concentration measured by FTIR, and calculated from a calibration performed at the same partial pressure and resolution. (3.65 $10^{34}$ molec. $\left.\mathrm{cm}^{-3}\right)$

Flux : lamp flux before experiment $\left(\int_{147 \mathrm{~nm}} \sim 10^{36}\right.$ photons. $\left.\mathrm{s}^{-1}\right)$

$\Phi_{\mathrm{HCN}}$ : quantum yield we want to estimate

$V$ : reactor's volume $\left(300 \mathrm{~cm}^{3}\right)$

$\mathrm{J}_{\mathrm{HCN}}$ : photolysis constant calculated from (Okabe, 1978) $\left(1.610^{-2} \mathrm{~s}^{-1}\right)$.

If a photostationary state is assumed $\frac{d[H C N]}{d t}=0$, then $\Phi_{H C N} \sim 0.2$.

The same calculation can be achieved for $\mathrm{NH}_{3}$, with $\left[\mathrm{NH}_{3}\right]=1.5810^{15}$ molec.cm ${ }^{-3}$ and $\mathrm{J}_{\mathrm{NH3}}=3.210^{-3} \mathrm{~s}^{-1}$. Then $\Phi_{N H 3} \sim 0.1$. Considering our hypothesis of a photostationnary state, if this equilibrium is not achieved in the reactor, the concentration of the studied product has not reached its stationary level and $[X]<[X]$ eq. Then our estimation is a lower limit.

Considering our second hypothesis, that the lamp flux is at its maximum during the irradiation, whereas it is decreasing because of the formation of a polymer on the window, we can derive that our estimation for the quantum yield is again a lower limit.

\section{GC-MS analysis}

GC-MS analysis after 6 hours irradiations of HMT at $147 \mathrm{~nm}$ is presented on Figure 5. Six products are detected. Compound 1 is not visible on the total mass plot (Figure 5-a), but it has been clearly identified to be $\mathrm{CH}_{3} \mathrm{CN}$ from a specific 
scan at 41 amu (Figure 5-b), its mass spectrum (Fig. 6 - Compound ${ }^{\circ} 1$ ), and its retention time measured after injection of the pure compound.
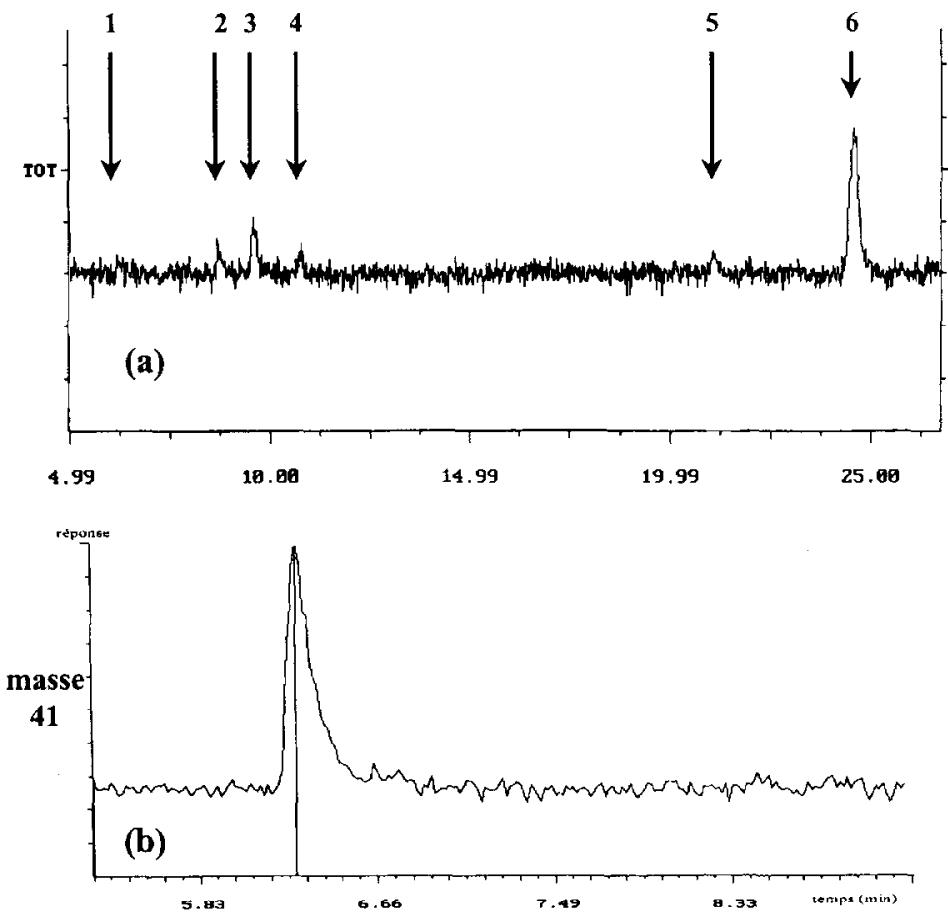

Figure 5: (a) Analysis of HMT $147 \mathrm{~nm}$ photodegradation products by GC-MS. Fig (b) is the specific chromatogram at mass 41 that lead to detection of $\mathrm{CH}_{3} \mathrm{CN}$, which is hidden in the noise of the total mass plot.

For compounds 2 to 6 , tentative identifications are proposed based on standard mass spectra from the NIST data base, or based on hypothesis concerning combinations between $\mathrm{C}, \mathrm{H}, \mathrm{N}$ and $\mathrm{O}$ atoms, and derived structures compatible with spectra. Our results are presented in table 1 . Experiments have also been performed at $122 \mathrm{~nm}$; only compounds 1 and 6 have been detected. This is certainly due to the low hydrogen lamp flux.

Table 1: Interpretation of chromatograms and mass spectra obtained after $\mathrm{HMT}+\mathrm{H}_{2} \mathrm{O}$ photolysis. ( $\mathrm{t}$ ) refers to a tentative detection.

\begin{tabular}{|c|c|c|c|c|}
\hline $\mathrm{II}^{\mathrm{O}}$ & Formula & Identification & $\begin{array}{l}147 \\
\mathrm{~nm}\end{array}$ & $\begin{array}{l}122 \\
\text { IIIII }\end{array}$ \\
\hline 1 & $\mathrm{CH}_{3} \mathrm{CN}$ & Retention Time & $\mathrm{x}$ & $\mathrm{X}$ \\
\hline $\begin{array}{c}2 \\
(\mathrm{t})\end{array}$ & $\begin{array}{c}\mathrm{CH}_{3} \mathrm{COOCH}_{2} \mathrm{CH}_{3} \\
\text { Ethylacetate } \\
\end{array}$ & NIST Mass Spectrum & $\mathrm{X}$ & \\
\hline $\begin{array}{c}3 \\
(\mathrm{t})\end{array}$ & $\begin{array}{c}\mathrm{CH}_{3} \mathrm{CH}_{2} \mathrm{CH}\left(\mathrm{CH}_{3}\right) \mathrm{CH}_{2} \mathrm{CH}_{2} \mathrm{CH}_{3} \\
\text { 3 Methyl, Hexane } \\
\end{array}$ & NIST Mass Spectrum & $\bar{X}$ & \\
\hline $\begin{array}{c}4 \\
(\mathrm{t})\end{array}$ & $\begin{array}{l}\mathrm{N} \equiv \mathrm{C}-\mathrm{CH}=\mathrm{CH}-\mathrm{C} \equiv \mathrm{N} \\
\text { 2, Butenedinitrile }\end{array}$ & $\begin{array}{c}78:(\mathrm{NCCHCHCN})^{+} \\
52:(\mathrm{NCCHCH})^{+} \\
39:(\mathrm{NCCHCHCN})^{2+}\end{array}$ & $\mathrm{X}$ & \\
\hline $\begin{array}{c}5 \\
(t)\end{array}$ & Not identified & Not identified & $\mathrm{x}$ & \\
\hline $\begin{array}{c}6 \\
(\mathrm{t})\end{array}$ & $\mathrm{N} \equiv \mathrm{C}-\mathrm{NH}-\mathrm{C} \equiv \mathrm{C}-\mathrm{C} \equiv \mathrm{N}$ & $\begin{array}{c}91:(\mathrm{NCNHCCCN})^{+} \\
65:(\mathrm{HNCCCN})^{+} \\
51:(\mathrm{HCCCN})^{+}\end{array}$ & $\mathrm{X}$ & $\mathrm{X}$ \\
\hline
\end{tabular}



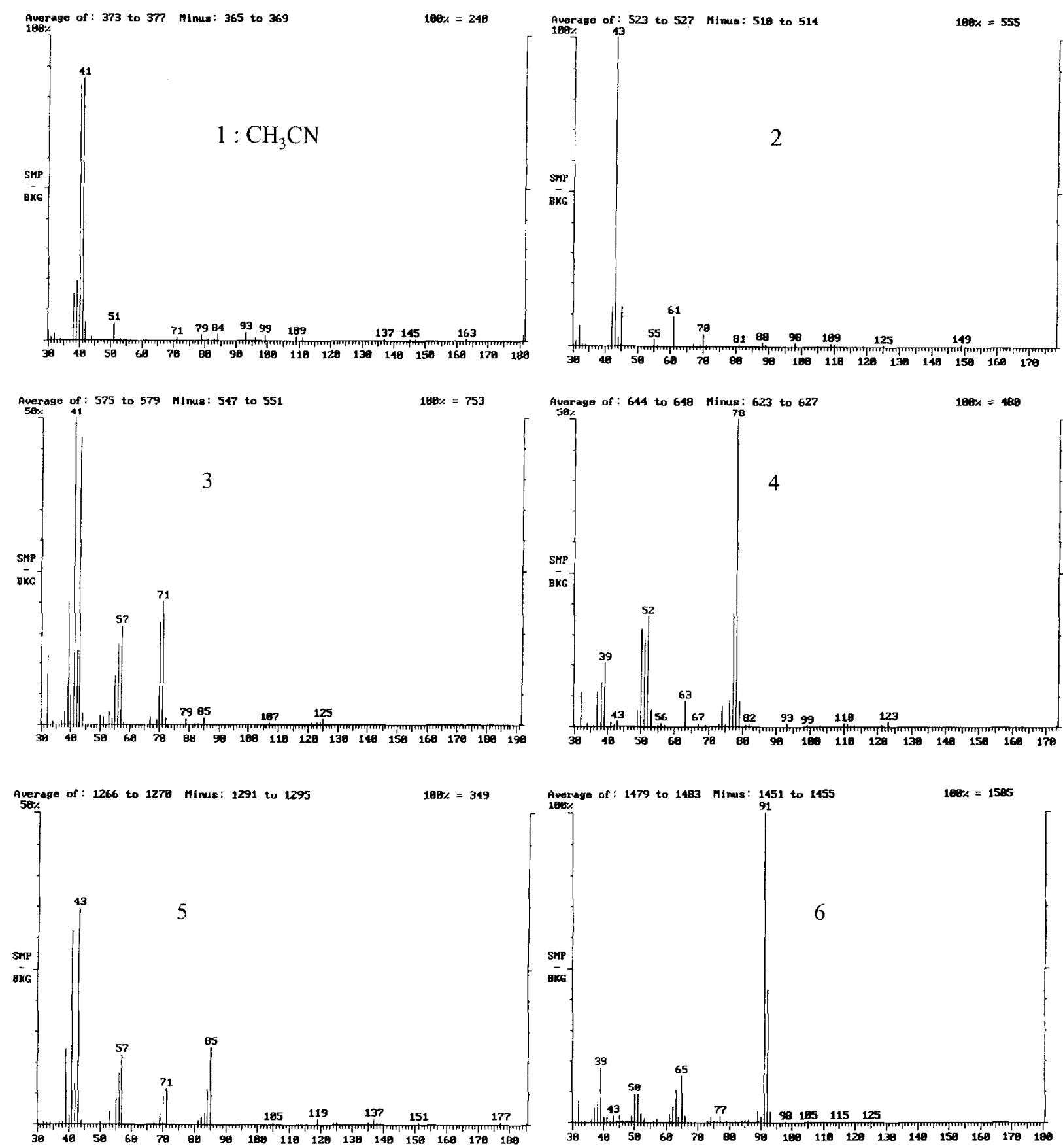

Fig. 6: Mass spectra of compounds 1 to 6 . Higher fragments than the parent ion are due to molecular recombinations in the ion trap.

\section{DISCUSSION}

Further analyses are required to confirm the detection of tentatively identified compounds, and to better constrain our quantitative data. This is a difficult task, mainly because of the « darkening » of the $\mathrm{MgF}_{2}$ window during irradiation, which shortens the possible duration of our experiments, and complicate achievement of quantitative results.

Nevertheless, those results show that the degradation of HMT is much more efficient in presence of water vapor: it yields more products, and induces quantum yield of about 0.2 and 0.1 for $\mathrm{HCN}$ and $\mathrm{NH}_{3}$ production, whereas it has been impossible to quantify HCN produced during photolysis under vacuum. It seems that the degradation process is activated by the photolysis products of water: $\mathrm{OH}^{\circ}, \mathrm{H}^{\circ}$. Those radicals may react with $\mathrm{HMT}$ and lead to its degradation. After breaking of the molecule, 
fragments may recombine. Dehydration of resulting compounds may lead to the formation of unsaturations observed in some of our resulting molecules.

Like (Bernstein et al., 1994), we detect nitriles and other $\mathrm{N}$ bearing molecules. But according to their results, a direct photolysis of HMT should be more efficient than we obtain. Indeed, they have shown a photodegradation of matrix-isolated HMT in Ar ice. Argon first ionization energy being of about $15 \mathrm{eV}$ (i.e. $\lambda \sim 82 \mathrm{~nm}$ ), HMT degradation in that matrix should be similar to our photolysis under vacuum as Ar is not involved in the degradation mechanism. Water traces in the argon matrix may be an explanation for this discrepancy.

Those data cannot yet be directly used in numerical models to interpret observation, because they have been obtained with a water pressure much higher than it can be encountered in cometary environment. A pressure dependence study is necessary to model the process, but it is likely that the use of lower water pressure will induce lower quantum yields. Nevertheless those preliminary results show that to understand the chemistry of comae, one have to consider the release of molecules into the gaseous phase from refractory material on grains.

Thus, if we think that a direct photodegradation of HMT on cometary grains cannot be a good explanation for observed $\mathrm{CN}$ and HCN extended sources, in the inner coma, interactions between water photoproducts, and outflnwing grains, may generate an additional source for these molecules: whether a direct production of $\mathrm{HCN}$, or a production of volatile nitriles that should be photodissociated to produce the observed CN. In this latter case, HMT would act as a grandparent molecule. For large scale extended sources, far from the nucleus, in regions where the density becomes too low to allow an efficient gasgrain interaction, a different molecule is required, which would released $\mathrm{CN}$ and $\mathrm{HCN}$ with a high yield by direct photodegradation under vacuum, or by thermal degradation. $\mathrm{HCN}$ polymers will soon be studied with our experimental set-up to address this question.

\section{REFERENCES}

Allamandola, L.J., S.A. Sandford, and G.J. Valero, Photochemical and thermal evolution of interstellar/precometary ice analogs, Icarus, 76, 225-252, 1988.

Bernstein, M.P., S.A. Sandford, L.J. Allamandola, and S. Chang, Infrared spectrum of matrix isolated Hexamethylenetetramine in $\mathrm{Ar}$ and $\mathrm{H} 2 \mathrm{O}$ at cryogenic temperatures., Journal of Physical Chemistry, 98, 12206-12210, 1994.

Bernstein, M.P., S.A. Sandford, L.J. Allamandola, S. Chang, and M.A. Scharberg, Organic Compounds Produced By Photolysis of Realistic Interstellar and Cometary Ice Analogs Containing Methanol, The Astrophysical Journal, 454, 327$344,1995$.

Biver, N., D. Bockelée-Morvan, J. Crovisier, J.K. Davies, H.E. Matthews, J.E. Wink, H. Rauer, P. Colom, W.R.F. Dent, D. Despois, R. Moreno, G. Paubert, D. Jewitt, and M. Senay, Spectroscopic Monitoring of Comet C/1996 B2 (Hyakutake) with the JCMT and IRAM Radio Telescopes, The Astronomical Journal, 118 (4), 1850-1872, 1999.

Bockelée-Morvan, D., J. Wink, D. Despois, N. Biver, P. Colom, J. Crovisier, E. Gerard, E. Lellouch, and L. Jorda, Interferometric imaging of molecular lines in comet Hale-Bopp, Bulletin of the American Astronomical Society, 30 (3), 31.02, 1998.

Briggs, R., G. Ertem, J.P. Ferris, J.M. Greenberg, P.J. McCain, C.X. Mendoza-Gomez, and W. Schutte, Comet Halley as an aggregate of interstellar dust and further evidence for the photochemical formation of organics in the interstellar medium, Origins of life and evolution of the biosphere, 22, 287-307, 1992.

Cottin, H., Chimie organique de l'environnement cométaire : étude expérimentale de la contribution de la composante organique réfractaire à la phase gazeuse, Doctorat thesis, Université Paris XII, Créteil, 1999. Available at: http://www.lisa.univ-paris12.fr/GPCOS/Hc/Hlt.htm

Cottin, H., M.C. Gazeau, Y. Bénilan, and F. Raulin, Polyoxymethylene as parent molecule for the formaldehyde extended source in comet Halley, The Astrophysical Journal, 556 (1), 417-420, 2001.

Cottin, H., M.C. Gazeau, J.F. Doussin, and F. Raulin, An experimental study of the photodegradation of polyoxymethylene at 122, 147 and $193 \mathrm{~nm}$, Journal of photochemistry and photobiology, 135 (A : Chemistry), 53-64, 2000.

Cottin, H., M.C. Gazeau, and F. Raulin, Cometary organic chemistry : a review from observations, numerical and experimental simulations, Planetary and Space Science, 47 (8-9), 1141-1162, 1999.

Crovisier, J., and F.P. Schloerb, The study of comets at radio wavelengths, in Comets in the Post Halley Era, edited by R.L.N.J.e. al., pp. 149-173, Kluwer, Dordrecht, 1991.

DiSanti, M.A., M.J. Mumma, N. DelloRusso, K. Magee-Sauer, R. Novak, and T.W. Rettig, Identification of two sources of carbon monoxide in comet Hale-Bopp, Nature, 399, 662-665, 1999.

DiSanti, M.A., M.J. Mumma, N.R.D. Russo, K. Magee-Sauer, R. Novak, T.W. Rettig, and M.N. Fomenkova, CO Emission in Comets C/1995 O1 (Hale-Bopp) and C/1996 B2 (Hyakutake): Evidence for a Distributed Source, Bulletin of the American Astronomical Society, 29, 34.02, 1997.

Eberhardt, P., D. Krankowsky, W. Schulte, U. Dolder, P. Lammerzahl, J.J. Berthelier, J. Woweries, U. Stubbeman, R.R. Hodges, J.H. Hoffman, and J.M. Illiano, The CO and N2 abundance in comet P/Halley, Astronomy and Astrophysic, 187, 481-484, 1987. 
Greenberg, J.M., What are comets made of ? A model based on interstellar dust, in Comets, edited by L.L. Wilkening, pp. 131163, University of Arizona Press, Tucson, 1982.

Kissel, J., and F.R. Krueger, The organic component in dust from comet Halley as mesured by the PUMA mass spectrometer on board Vega 1, Nature, 326, 755-760, 1987.

Klavetter, J.J., and M.F. A'Hearn, An extended source for CN jets in Comet P/Halley, Icarus, 107 (2), 322-334, 1994.

Lis, D.C., J. Keene, K. Young, T.G. Phillips, D. Bockelée-Morvan, J. Crovisier, P. Schilke, P.F. Goldsmith, and E.A. Bergin, Spectroscopic Observations of Comet C/1996 B2 (Hyakutake) with the Caltech Submillimeter Observatory, Icarus, 130 (2), 355-372, 1997.

Meier, R., P. Eberhardt, D. Krankowsky, and R.R. Hodges, The extended formaldehyde source in comet P/Halley, Astronomy and Astrophysics, 277, 677-691, 1993.

Mitchell, D.L., R.P. Lin, C.W. Carlson, A. Korth, H. Rème, and D.A. Mendis, The origin of complex organic ions in the coma of comet Halley, Icarus, 98, 125-133, 1992.

Okabe, H., Photochemistry of small molecules, Wiley-Interscience Publication, 1978.

Rauer, H., C. Arpigny, H. Boehnhardt, F. Colas, J. Crovisier, L. Jorda, M. Küppers, J. Manfroid, K. Rembor, and N. Thomas, Optical Observations of Comet Hale-Bopp (C/1995 O1) at Large Heliocentric Distances Before Perihelion, Science, 275, 1909-1912, 1997.

Schutte, W.A., L.J. Allamandola, and S.A. Sandford, An Experimental Study of the Organic Molecules Produced in Cometary and Interstellar Ice Analogs by Thermal Formaldehyde Reactions, Icarus, 104, 118-137, 1993a.

Schutte, W.A., L.J. Allamandola, and S.A. Sandford, Formaldehyde and organic molecule production in astrophysical ices at cryogenic temperatures, Science, 259, 1143-1145, $1993 \mathrm{~b}$.

Veal, J.M., L.E. Snyder, M. Wrigth, L.M. Woodney, P. Palmer, J.R. Forster, I.d. Pater, M.F. A'Hearn, and Y.J. Kuan, An Interferometric Study of HCN in Comet Hale-Bopp (C/1995 O1), The Astronomical Journal, 119 (3), 1498-1511, 2000.

Wolman, Y., S.L. Miller, J. Ibanez, and J. Oro, Science, 174, 1039, 1971.

Woodney, L.M., M.F. A'Hearn, D.D. Wellnitz, D.G. Schleicher, T.L. Farnham, T.C. Cheung, J.P. McMullin, J.M. Veal, L.E. Snyder, I.D. Pater, J.R. Forster, M.C.H. Wright, P. Palmer, Y.J. Kuan, and N.H. Samarasinha, Morphology of HCN and $\mathrm{CN}$ in Comet Hale-Bopp, Bulletin of the American Astronomical Society, 30, 31.03, 1998. 